\title{
A Novel design of Antenna for biosensing applications
}

\author{
Fu-Chieh Chang ${ }^{1}$, Jerome Shiu' ${ }^{2}$ You-Zheng Yin ${ }^{1}$, Chii-Wann Lin ${ }^{1,2,3^{*}}$ \\ ${ }^{1}$ Institute of Biomedical Engineering, National Taiwan University, Taipei, Taiwan (10617) \\ ${ }^{2}$ Institute of Bioelectronics and Bioinformatics, National Taiwan University, Taipei, Taiwan (10617) \\ ${ }^{3}$ Institute of Applied Mechanics, National Taiwan University, Taipei, Taiwan (10617) \\ *Corresponding author: cwlinx@ntu.edu.tw
}

\begin{abstract}
:
An antenna-based sensor with novel combinational design of metamaterial and transmission line are proposed for the applications of biosensors. Working at $1.6 \mathrm{GHz}$ resonant frequency, our design aims to reduce the size of antenna biosensor by applying metamaterial structure. The active sensing area of antenna was optimized based on the distribution of electrical field to measure the permittivity and loss tangent of sample by the scattering parameters. The resonant frequency shifts from $1.69 \mathrm{GHz}$ to $1.63 \mathrm{GHz}$ under different concentrations of water-ethanol solutions (3\% to $100 \%$ ) with equivalent permittivity of 79 to 14 . According to our results, this antenna biosensor has advantages of small size, lower resonant frequency, real time, label free, sensitive, low profile and easy to integrated with printed circuit boards (PCB) for practical applications.
\end{abstract}

Key words: metamaterial, antenna biosensor

\section{Introduction}

Antenna biosensors have several advantages. For example, it provides a real-time detection of the electric or magnetic characteristics of materials at radio frequency without the needs of labelling, chemical modification, or physical intrusion. Hence, it has drawn intensive interests in the research fields of material science and biological research [1]. The sensing principle is to detect the changes of the relative permittivity $\left(\varepsilon_{r}\right)$, and loss tangent $(\tan \delta)$ in the resonant band and the return loss $\left|S_{11}\right|$ level caused by the samples. However, it is a trade off between the designed antennas size and the resonant wavelength in general. The development trends of biosensing devices are toward smaller size, non-invasive way, and integration with microfluidic devices for sample handling. Antennabased biosensor needs to answer all these challenges as well. Hence, we adapted metamaterial idea in antenna design to reduce the dimension of this sensor [2] in steady of working toward higher resonant frequency. Moreover, the sensing location on this antenna chip has been optimized based on the simulation result of designed antenna structure. Finally, various concentrations of ethanol solutions were measured to demonstrate to sensor performance.

\section{Methods}

The analysis of the antenna biosensor is based on the theory of microstrip transmission line
(TL), which assumes that most of the energy is concentrated in the space between the microstrip line and the ground plane. We designed a slot on the backside of antenna to provide a constant electric flux (near field) that is used to detect the samples, and the other part of electric field is shielded by copper plate as shown in Fig. 1. The slot which acts as a probe, is pressed against the test specimen. Thus, without directly attaching to the feed line this measurement method offers a better isolation between the feed and the samples. Also, it can overcome the limitations of spatial resolution and which can obtain enough accuracy with same consequent in every experiment. [4].

The geometry and dimensions of the antenna biosensor are described in detail in the caption of Fig. 2. The circuit design of antenna follows the concept of the composite right/left-handed (CRLH) metamaterial [3]. The antenna biosensor's left-handed (LH) capacitance (CL) is attributed to the interdigital capacitors coupling between the patch, and antenna biosensor's LH inductance (LL) is due to the shorted-stub inductances to ground. The right-handed (RH) effects are due to the capacitive (CR) coupling between the patch and ground plane, and the current flow atop the patch (LR). Fig. 3 shows 


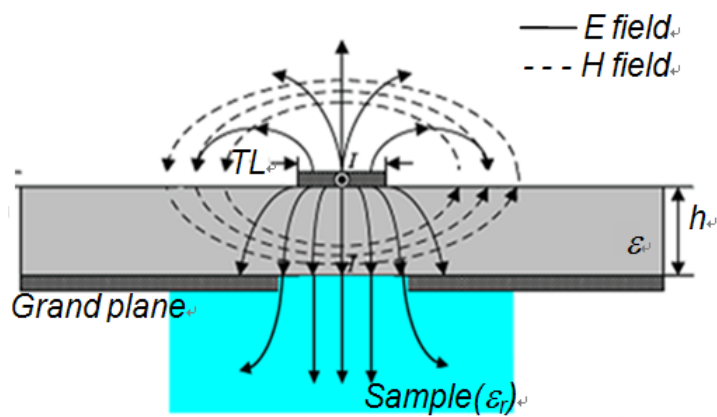

Fig. 1. The electric and magnetic field distribution on both sides of the microstrip transmission line.

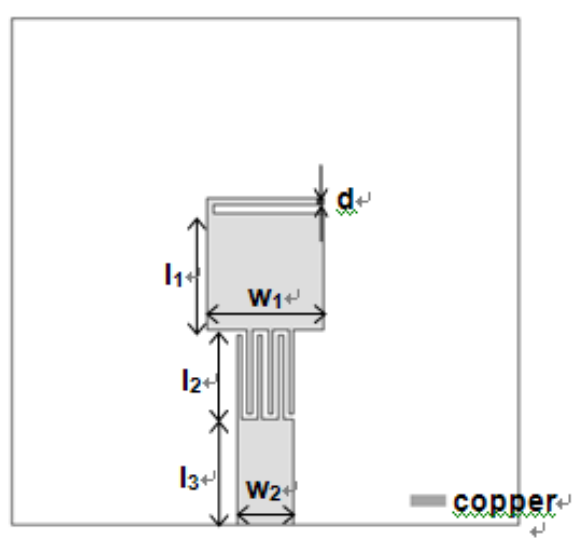

(a) The layout on the top side

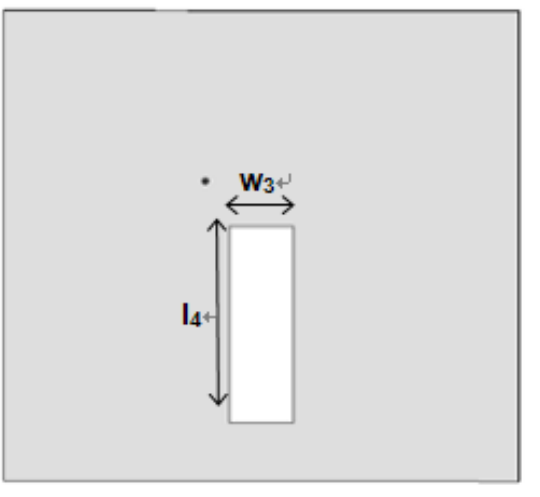

(b) The layout on the back side

Fig. 2. The dimensions of the designed antenna biosensor element (a) on the top are $d=0.3 \mathrm{~mm}, I_{1}=$ $w_{1}=5.5 \mathrm{~mm}, I_{2}=4 \mathrm{~mm}, I_{3}=5 \mathrm{~mm}, w_{2}=2.7 \mathrm{~mm}$, respectively.(b) Also, the bottom of slot dimensions are $w_{3}=3 \mathrm{~mm}$ and $I_{4}=10 \mathrm{~mm}$.

the proposed antenna-based biosensor is designed and fabricated by using the FR4 substrate on printed circuit board (PCB) having a double-faced copper film with electrical parameters of permittivity $\varepsilon_{r}=4.4$, loss tangent $\tan \delta=0.02$ and thickness $\mathrm{h}=0.8$. All simulations in this work were carried out using Ansoft HFSS 12.0. The measurement was carried out by a RF network analyzer (Agilent E5071B).
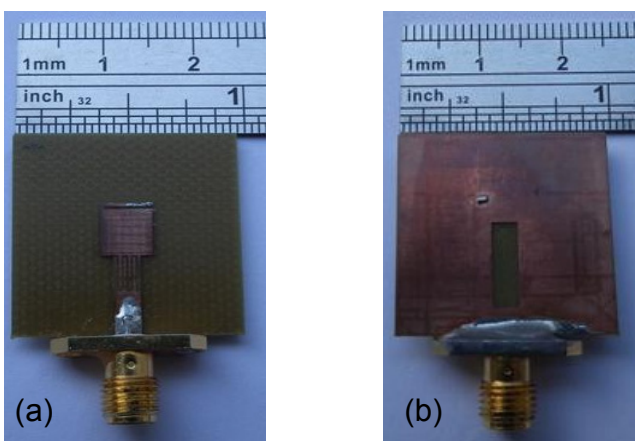

Fig. 3. Picture of the biosensing antenna.(a)top view (b)bottom view.

For the proposed antenna biosensor, the resonant frequency and return loss can be calculated by the following equation

$$
\begin{aligned}
f r & =\frac{1}{2 \pi \sqrt{L C}} \\
C & =\varepsilon_{\text {eff }} \frac{A}{d}
\end{aligned}
$$

, where $\varepsilon_{\text {eff }}$ is the effective permittivity. In our case, this value is the average permittivity across the PCB substrate and the sample under test. The effective permittivity comprised of real and imaginary components as following equation:

$\varepsilon_{\text {eff }}=\varepsilon_{\text {eff }}{ }^{\prime}-j \varepsilon_{\text {eff }} "$

, where $\varepsilon_{\text {eff }}{ }^{\prime}$ gives rise to electromagnetic transmittance through lossless reaction, and $\varepsilon_{\text {eff }}$ "signifies transmittance through energy loss reaction. [5,6] The ratio of the $\varepsilon_{\text {eff }}$ " to $\varepsilon_{\text {eff }}$ ' is defined as loss tangent $(\tan \delta)$, which measures the electromagnetic wave energy loss upon transmittance:

$$
\tan \delta » \frac{\varepsilon_{\text {eff" }} "}{\varepsilon_{\text {eff }}^{\prime}}
$$

Experimentally, resonance frequency shift indicate the change in $\varepsilon_{\text {eff. }}$ The change in $\left|S_{11}\right|$ measures the $\tan \delta$ of the sample[7]. For the permittivity and loss tangent measurement of liquid materials, the specimens were placed on the bottom of the slot. To validate the availability of this design, full wave 3D simulations have been performed on the two parameters of permittivity and loss tangent. The results are shown in Fig. 4 (a) and (b), respectively. Similar to theoretical prediction, the permittivity inducing the shifts of frequency and $\left|S_{11}\right|$ level at the resonant frequency of $1.6 \mathrm{GHz}$, and the loss tangent only has information of $\left|S_{11}\right|$ level shift. 

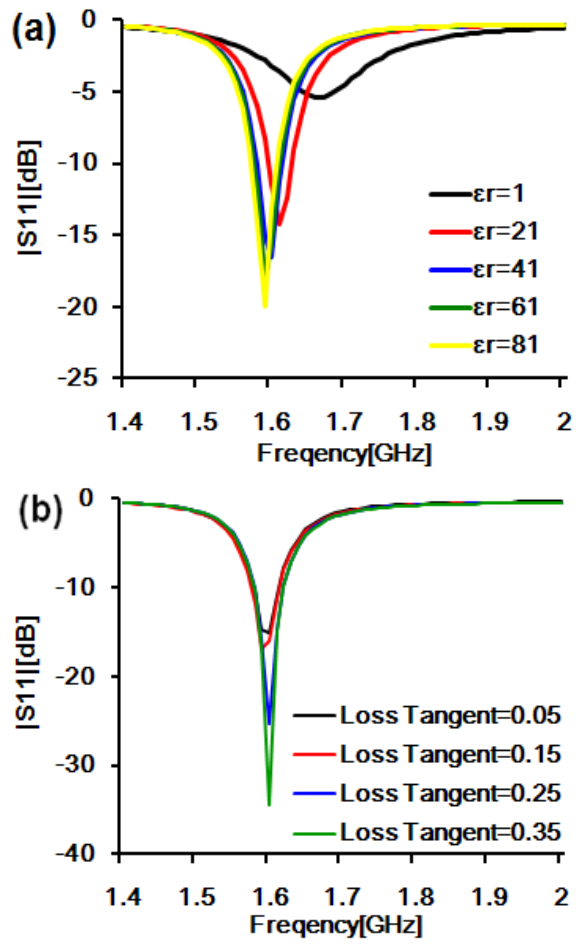

Fig. 4. Simulation result. (a) The relationship of frequency versus the permittivity. (b) The relationship of frequency versus the permittivity

\section{Results and Discussion}

For the liquid material permittivity and loss tangent measurements, the sample under test was positioned on the bottom of the slot. Before measuring the permittivity of unknown samples it is essential to test the biosensor by measuring samples with a known permittivity. There are two references which can be used as standards before measuring other specimens. The permittivity of air is 1 , and de-ionized water is 79.[8] The corresponding measurements are shown in Fig. 5.

To demonstrate the capability for bio-sensor, the relationship of equivalent permittivity and curve of loss tangent versus various concentrations of ethanol and methanol are shown in Fig. 6 and Fig. 7, respectively. We show the changes of $\left|S_{11}\right|$ and resonant frequency caused by the ethanol-water and the methanol-water mixtures with concentrations of $3.125 \%, 6.25 \%$, $12.5 \%, 25 \%, 50 \%$, and $100 \%$. This antennabased biosensor shows the potential to identify the different concentrations of liquid solution. In addition, Fig. 8 shows both methanol and ethanol get almost the same effective permittivity and resonant frequency in the same concentration, but it still can distinguish different substances between ethanol and methanol through the $\tan \delta$. However, this biosensing antenna cannot effectively distinguish for low concentration of the samples. The resonance may be the

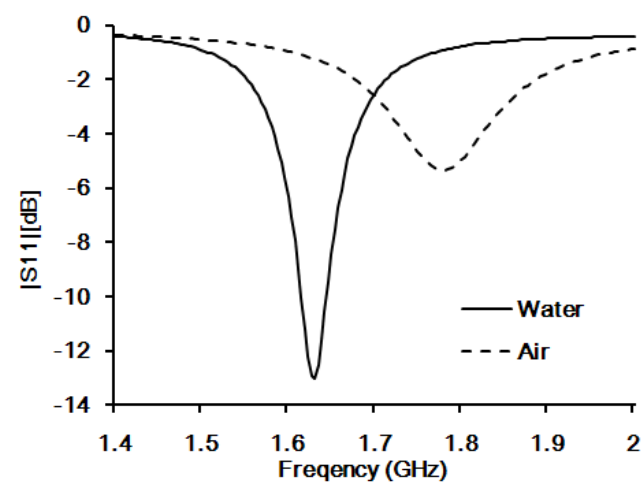

Fig. 5. The spectra of $|S 11|$ of water and air
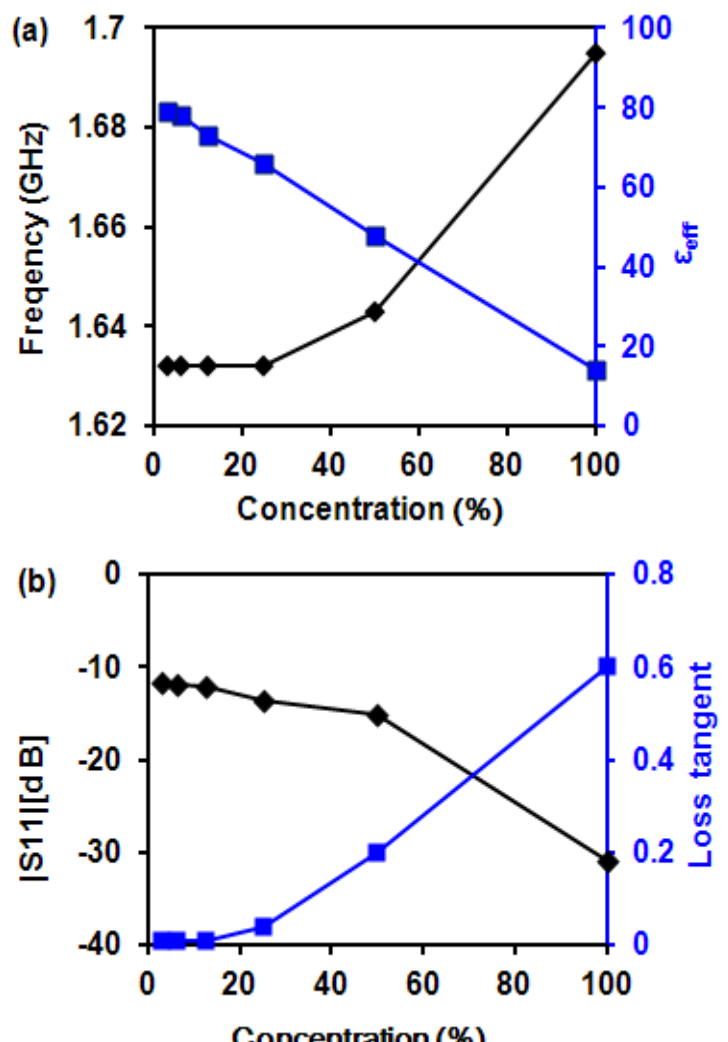

Fig. 6. Resonant frequency and (b) $|S 11|$ caused by the different concentrations of Ethanol.

thickness of the PCB substrate. To improve the sensitivity, a thinner PCB can be used to design the biosensing antenna.

\section{Conclusion}

In this work, we have successfully demonstrated the development of a metamaterial antennabased biosensor for biochemcial sensor and novel measurement mode for quick and accurate analysis of the specimen. This device also offers advantages of cost effective due to the fabrication with $\mathrm{PCB}$ process and real time measurement with label-free samples. In the future, the thickness of device will be improved for detection of low concentration and smaller sample volume. 

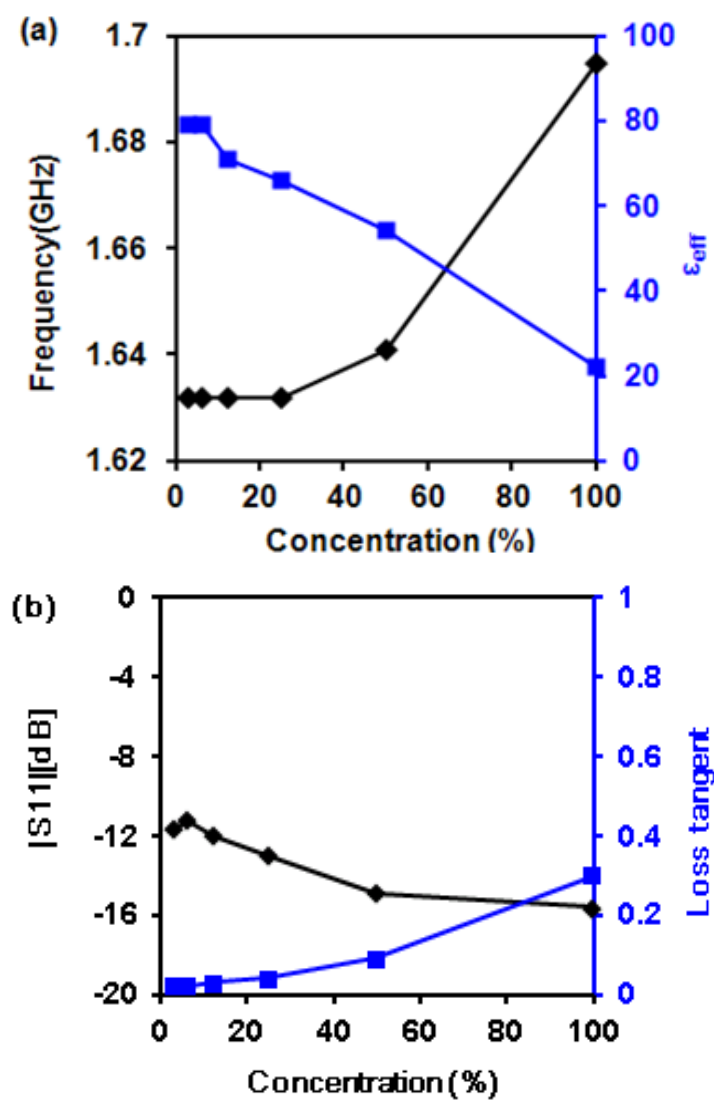

Fig. 7 (a) Resonant frequency and (b) |S11| caused by the different concentrations of Methanol.

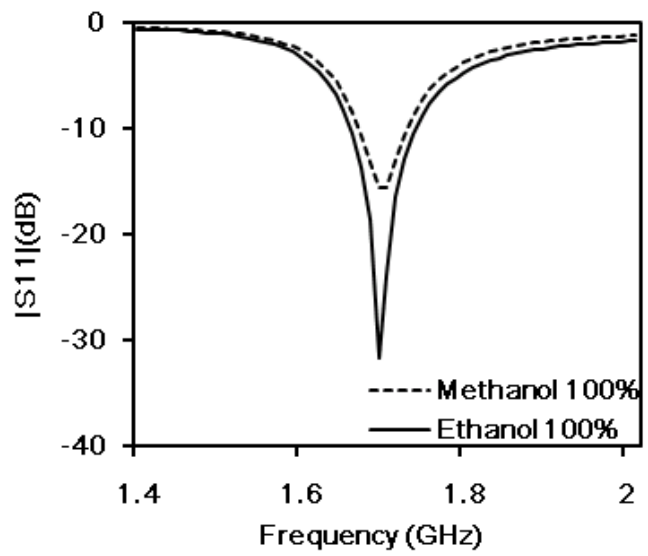

Fig. 8 The spectra of $|S 11|$ of $100 \%$ methanol and $100 \%$ ethanol

\section{References}

[1] A. P. Gregory and R. N. Clarke, A review of RF and microwave techniques for dielectric measurements on polar liquids, IEEE Trans. Dielectr. Electr. Insul. 13, 727-743 (2006); doi: 10.1109/TDEI.2006.1667730

[2] C. Caloz and T. Itoh, Electromagnetic metamaterials: transmission line theory and microwave applications: Wiley-IEEE Press, (2006).
[3] Caloz, C.; , Dual Composite Right/Left-Handed (D-CRLH) Transmission Line Metamaterial, Microwave and Wireless Components Letters 16, 585-587 (2006);

doi: 10.1109/LMWC.2006.884773

[4] Akhavan, H.G.; Mirshekar-Syahkal, D.; , Slot antennas for measurement of properties of dielectrics at microwave frequencies, Antennas and Propagation, 8-11(1999) doi:10.1049/cp:19990003

[5] D. Pozar, Microwave Engineering, Wiley, Hoboken, (2005).

[6] Taylor, K.M.; van der Weide, D.W.; Ultrasensitive detection of protein thermal unfolding and refolding using near-zone microwaves, Microwave Theory and Techniques 53, 1576- 1586, (2005); doi: 10.1109/TMTT.2005.847047

[7] Wiwatcharagoses, N.; Kyoung Youl Park; Hejase, J.A.; Williamson, L.; Chahal, P.; , Microwave artificially structured periodic media microfluidic sensor, Electronic Components and Technology Conference, 1889-1893 (2011) doi: 10.1109/ECTC.2011.5898773

[8] W.J. Ellison, K. Lamkaouchi, J.-M. Moreau, Water: a dielectric reference, Journal of Molecular Liquids 68, 171-279 (1996), doi:10.1016/0167-7322(96)00926-9. 